Himat Vaghadia MB FRCPC, Leonard C. Jenkins BA MD CM FRCPC

\title{
Use of a Doppler ultra- sound stethoscope for intercostal nerve block
}

Two cases with rib injuries are reported where intercostal nerve block without rib palpation was safely and successfully performed on six separate occasions using a Doppler blood-flow detector ultrasound stethoscope. A third case studied by a radiologist using a pulsed Doppler flowmeter, determined the source of the Doppler signals as originating from the intercostal artery. The significance of these findings is discussed.

The technique of intercostal nerve block is well established. ${ }^{12}$ It is particularly useful for analgesia after rib fractures. However, the traditional technique suffers the major disadvantage that preliminary rib palpation is required for accurate placement of the local anaesthetic. ${ }^{1}$ Rib palpation may not be possible in some patients because of pain and swelling of the overlying tissues. Any technique that permits intercostal nerve block without rib palpation in such patients would be an advantage. Two cases are presented where a Doppler ultrasound stethoscope was used to perform intercostal block without rib palpation.

\section{Case reports}

\section{Case 1}

A 23-year-old $53 \mathrm{~kg}$ woman, height $159 \mathrm{~cm}$, presented with undisplaced fractures of the left eighth and ninth ribs in the posterior axillary line sustained during a skiing accident. Her medical history was unremarkable and there was no evidence of a pneumothorax on the chest roentgenogram.

\section{Key words}

ANAESTHESIA: regional; TECHNIQUE: intercostal nerve block; EQUIPMENT: Doppler ultrasound stethoscope.

From the Department of Anaesthesia, Faculty of Medicine, The University of British Columbia, Vancouver General Hospital, Vancouver, British Columbia.

Address correspondence to: Dr. H. Vaghadia, Department of Anaesthesia, Faculty of Medicine, The University of British Columbia, 910 West 10th Avenue, Vancouver, B.C., V5Z 4E3.
Meperidine $50 \mathrm{mg}$ IM was prescribed for analgesia but she still complained of pain. Tenderness over the lower left chest also prevented rib palpation. The patient agreed to the use of the indirect method of intercostal nerve block using a Doppler ultrasound stethoscope (Figure 1). She was placed prone and ultrasound transmission gel was applied to the skin along the lateral edge of the erector spinae muscle on the left side. A Doppler stethoscope probe was placed in contact with the skin about $7-8 \mathrm{~cm}$ from the midline, approximately at the level of the seventh rib. The probe was then gently manipulated up and down along the long axis over a distance of $1-2 \mathrm{~cm}$, until intercostal arterial flow became audible as a pulsatile pumping sound. The position of maximum signal intensity was marked. A $25 \mathrm{G}$ needle, $5 \mathrm{~cm}$ long, was inserted aseptically at this site with its point angled slightly cephalad to make contact with the seventh rib. Bupivacaine 0.5 per cent solution $(3 \mathrm{ml}$ ) with epinephrine 1:200000 was injected after "crawling" the needle to the inferior border of the rib in the usual manner, ${ }^{1,2}$ The Doppler stethoscope was used to facilitate identification of the left eighth, ninth and tenth ribs and $3 \mathrm{ml}$ of the local anacsthetic solution were injected at each site. The procedure was completed within ten minutes and complete pain relief was produced. This technique was repeated on a further two occasions and chest roentgenograms taken after each occasion confirmed the absence of a pneumothorax.

\section{Case 2}

A 46-year-old $96 \mathrm{~kg}$ man, height $168 \mathrm{~cm}$, presented with undisplaced fractures of the right sixth and seventh ribs in the mid-axillary line sustained during a motor vehicle accident. History and examination were non-contributory and a pneumothorax was absent on the chest roentgenogram. Intercostal block wia the traditional method ${ }^{1,2}$ could not be performed because rib palpation was prevented by pain and swelling of the overlying tissues. A Doppler stethoscope was successfully used to perform intercostal block without rib palpation on three occasions using the technique described above for patient 1 . Bupivacaine 0.5 per cent ( $3 \mathrm{ml}$ per intercostal nerve) with epinephrine 1:200000 was used to anaesthetise the right fifth, sixth. seventh and eighth intercostal nerves and resulted in complete pain relief. This technique was repeated on a 


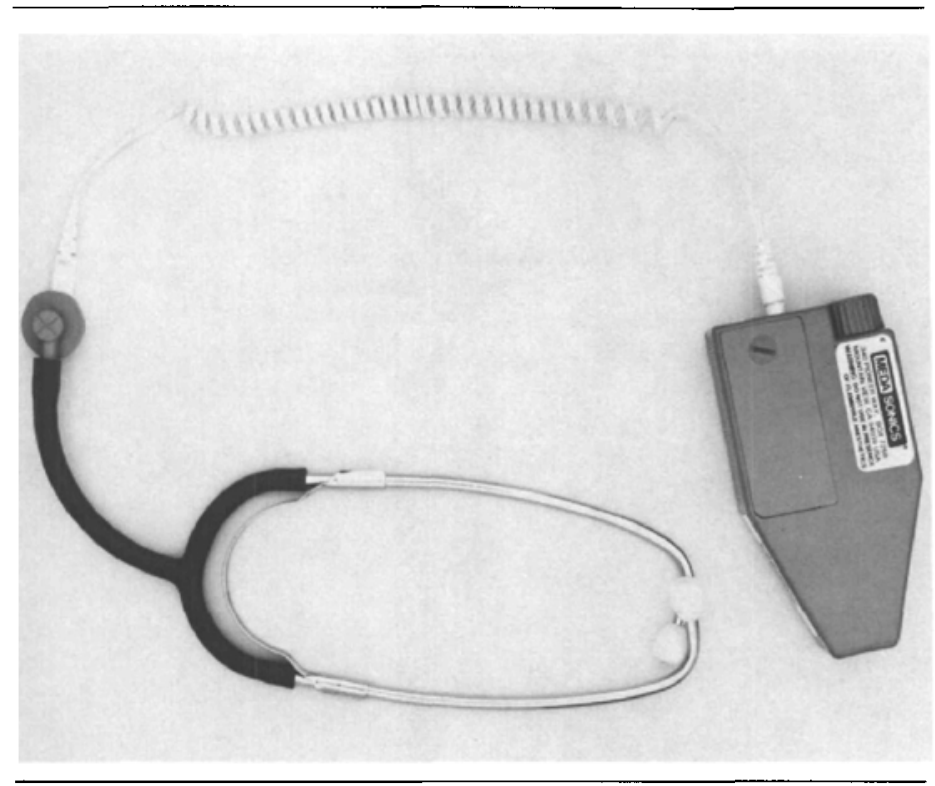

FIGURE I Doppler blood-flow detector ultrasound stethoscope operating at $5.3 \mathrm{MHz}$ (Model BF4A, Medasonics, Mountain View, California, U.S.A.).

further two occasions. Chest roentgenograms taken after each occasion confirmed the absence of a pneumothorax.

\section{Case 3}

A 32-year-old $70 \mathrm{~kg}$ man, height $175 \mathrm{~cm}$, was studied in order to determine whether signals obtained with a Doppler stethoscope were reproducible with a more sophisticated pulsed Doppler flowmeter and to determine the source of these signals. He was placed prone and ultrasound transmission gel applied to the skin along the lateral edge of the erector spinae muscle on both sides. A Doppler stethoscope probe was placed in contact with the skin about $7 \mathrm{~cm}$ from the midline approximately at the level of the left eighth rib. The probe was manipulated as described for patient 1 until intercostal arterial flow became audible. The position of maximal arterial flow signal was marked. A radiologist was then asked to evaluate the patient with a pulsed Doppler flowmeter (Diasonics 400, Diasonics Inc, Malpitas, Califomia, U.S.A.) operating at a frequency of $4.5 \mathrm{MHz}$. The position of maximum signal intensity was identical to that obtained with the Doppler stethoscope. The source of these arterial flow signals was identified as the intercostal artery (Figure 2) lying at a depth of about $2 \mathrm{~cm}$ from the skin surface. Similar agreement between the Doppler ultrasound stethoscope and the pulsed Doppler flowmeter were obtained for the right eighth rib and intercostal artery.

\section{Discussion}

Posterior intercostal block is usually performed about $7 \mathrm{~cm}$ from the midline near the edge of the erector spinae muscle. ${ }^{1,2}$ Anatomical studies in cadavers have shown a variability in the arrangement of intercostal arteries and nerves in relation to the inferior border of the rib at this location. ${ }^{3,4}$ In many patients the intercostal artery lies just along the inferior edge of the rib with the intercostal nerve either above or below it. ${ }^{3}$ Thus, a Doppler stethoscope located over the inferior edge of the rib should be able to detect blood flow signals from the intercostal artery. This hypothesis was confirmed in Cases 1 and 2 with respect to ribs 5-8 (Case 2) and ribs 7-10 (Case 1). In addition, studies in Case 3 confirmed the source of these signals to be consistent with the position of the intercostal artery, as demonstrated by previous anatomical studies, ${ }^{3}$

When an intercostal artery occupies a more superior position behind the inferior border of a rib, ${ }^{3}$ a Doppler stethoscope located just below the inferior rib border with its probe angulated cephalad should be able to detect the arterial flow signal. Experience with the above three cases suggests that an optimal approach might involve placement of the Doppler stethoscope probe approximately 


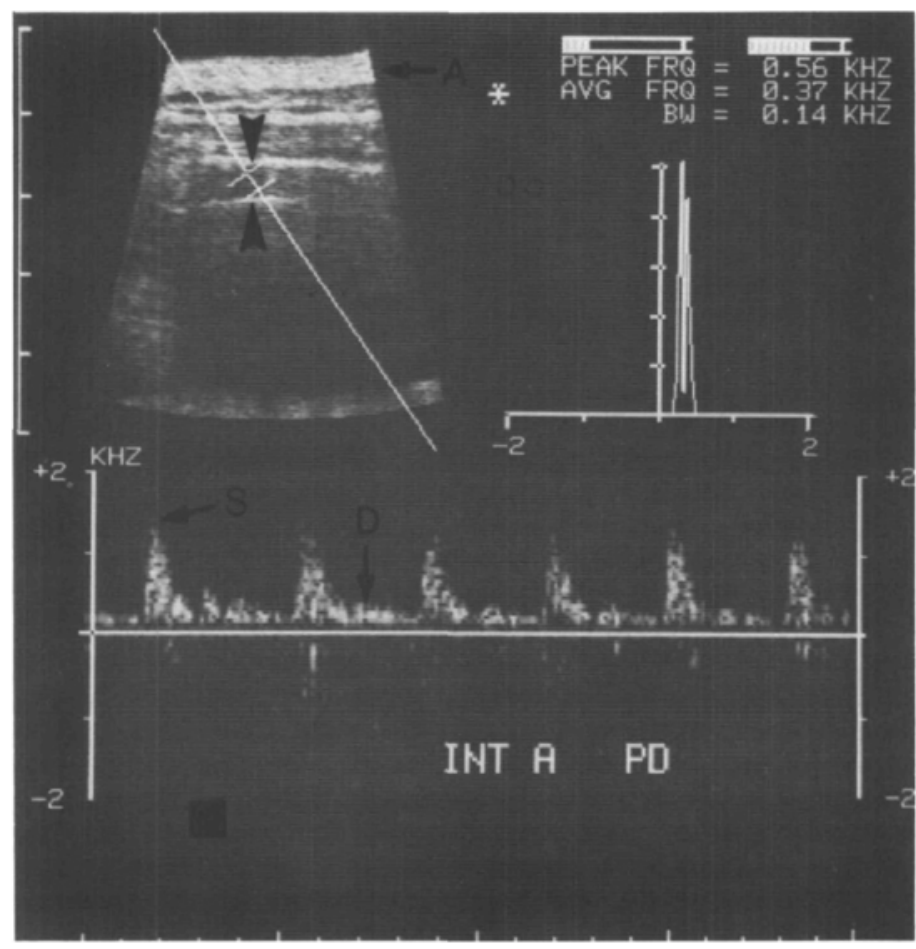

FIGURE 2 Pulsed Doppler-flow signals from the eighth intercostal artery. Inset shows image of the artery lying bencath the skin surface (between artowheads). $A=$ skin and subcutaneaus tissue; $S=$ systolic anterial flow; $\mathrm{D}=$ diastolic arterial flow.

over the position where intercostal block is to be performed. Subsequent movement of the instrument probe slowly up and down by $1-2 \mathrm{~cm}$ while tilting its axis so that the probe points cephalad, should be able to identify an intercostal artery that is "hidden"' behind the rib. Furthermore, use of the Doppler stethoscope $7 \mathrm{~cm}$ from the midline ensures that flow signals from the descending aorta and inferior vena cave do not cause interference. Doppler flowmeters have been used in the past for detecting flow signals from very small vessels such as branches of mammary arteries ${ }^{5}$ and digital vessels ${ }^{6}$ in the hand. Intercostal arteries are considerably larger in diameter and the flow signal generated is easily audible. The important factor is that the Doppler device should operate at a frequency that allows detection of blood flow with minimal signal attenuation by the more superficial tissues. As the frequency of a Doppler device increases, the rate of signal attenuation also increases. For small, relatively superficial vessels such as the intercostal arteries, Doppler devices operating at $4-10 \mathrm{MHz}$ should be adequate as they will be able to detect blood flow signals to a depth of about $5 \mathrm{~cm} .^{7}$ In the average individual, intercostal arteries lie at a depth of about $2.5 \mathrm{~cm}$ beneath the skin near the lateral edge of erector spinae muscle. ${ }^{3}$ Use of a Doppler stethoscope for identifying intercostal arteries and hence the inferior border of the ribs during performance of an intercostal block was not revealed in a computerized search of the literature back to 1966. The procedure was hazard free in the two patients described. In addition, detection of arterial flow signals did not require firm application of the Doppler probe to the skin and was therefore not associated with pain. A possible disadvantage may be failure to detect an intercostal artery that is completely hidden behind the superior edge of a rib. Such an anatomical arrangement has not been described. ${ }^{3,4}$

In conclusion, the use of a Doppler blood-flow detector ultrasound stethoscope for performing intercostal nerve block without rib palpation is described. The technique was easy and reliable. It may prove useful in patients 
where rib palpation is prevented by pain or swelling; it may also have application in obese individuals and as a teaching aid in instructing the beginner in intercostal block techniques, the applied anatomy of the area, and thus merits further attention.

\section{Acknowledgements}

The authors thank Dr. A. Buckley, Department of Radiology and Ultrasound, Vancouver General Hospital, for help with this report.

\section{References}

1 Moore $D C$, Bridenbaugh $L D$. Intercostal nerve block in 4,333 paticnts. Indications, techniques and complications. Anesth Analg 1962; 41: 1-23.

2 Murphy TM. Nerve Blocks. In: Miller RD (ed). Anaesthesia. New York: Churchill Livingstone, 1986: 1015.

3 Nunn JF, Slavin G. Posterinr intercostal block for pain relief after cholecystectomy. Anatomical basis and efficacy. Br J Anaesth 1980; 52: 253.

4 Moore $D C$. Anatomy of the intercostal nerve. Its importance during thoracic surgery. Am J Surg 1982; 144: 371.

5 Aoyagi $F$, Fujino $T$, Ohshiro $T$. Detection of small vessels for microsurgery by Doppler flowmeter. Plastic and Reconstructive Surg 1975; 55: 372 .

6 Pearce WH, Ricco J, Yao JST. Upper extremity vascular diagnosis using Doppler Ultrasound. In; Zwiebel WJ (ed). Introduction to Vascular Ultrasonography. New York: Grune and Stratton 1986: 259

7 Zagzebski JA. Physics and instrumentation in Doppler and B-Mode Ultrasonography. In: Introduction to Vascular Ultrasonography Zwiebel WI (ed). New York: Grune and Stratton 1986: 21 .

\section{Résumé}

Deux cas de fractures de cótes sont rapportés où un bloc intercostal sans palpation des cótes a été effectué avec succès six fois à des occasions séparées utilisant un stéthoscope d ultrason détectant le flot sanguin par Doppler. Un troisième cas étudié par un radiologiste utilisant un débimètre pulsé Doppler a déterminé que la source de signaux Doppler était l'artère intercostal. La signification de ces trouvailles est discutée. 